\title{
The role of alpha2 agonists in pediatric anesthesia
}

\author{
Gregory B. Hammer MD
}

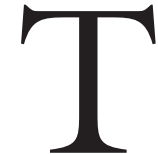

HE alpha2-receptors are involved in regulating the autonomic and cardiovascular systems. Alpha2-receptors are located on blood vessels, where they mediate vasoconstriction, and on sympathetic terminals, where they inhibit norepinephrine release. Alpha2-receptors also are located within the central nervous system, and their activation leads to sedation, a reduction of tonic levels of sympathetic outflow and catecholamines, and an augmentation of cardiac-vagal activity. In addition, alpha2-receptors within the spinal cord modulate pain pathways, thereby providing some degree of analgesia.

Alpha2-induced sedation qualitatively resembles normal sleep; specifically, the endogenous, nonrapid eye movement, sleep-promoting pathways may mediate alpha2-induced sedation. ${ }^{1}$ Endogenous sleep pathways are causally involved in dexmedetomidine-induced sedation. Thus, the participation of nonrapid eye movement sleep pathways seems to explain why patients who appear to be "deeply asleep" from dexmedetomidine are relatively easily aroused in much the same way as occurs with natural sleep.

The respiratory sparing effects of alpha2-agonists have been closely examined. They are associated with minimal respiratory depression and a preservation of the ventilatory response to $\mathrm{CO}_{2}$. In healthy volunteers, plasma levels of dexmedetomidine eightfold to tenfold higher than the clinical range resulted in unarousable volunteers who did not respond to a painful stimulus but maintained their respiratory drive. The $\mathrm{PaCO}_{2}$ increased from a baseline of 43 $\mathrm{mmHg}$ to only $47 \mathrm{mmHg}{ }^{2}$

In high doses, alpha2-agonists have the potential for causing both systemic and pulmonary hypertension and direct or reflex bradycardia. In the lower, clinical range, alpha2-agonist infusions decrease blood pressure, presumably as a result of a central sympatholytic effect. At higher plasma levels, peripheral alpha2-receptor mediated vasoconstriction overrides the sympatholytic effects, resulting in increased pulmonary artery and systemic blood pressures. In healthy patients with intact baroreflexes, this can lead to bradycardia. Reflex bradycardia in conjunction with the vagal mimetic property of alpha2-agonists could lead to severe bradycardia or asystole.

Dexmedetomidine is the pharmacologically active dextroisomer of medetomidine. It was approved by the US Food and Drug Administration in 1999 as a short-term medication $(<24 \mathrm{hr})$ for sedation of patients in the intensive care unit, and it has also been shown to act as a general anesthetic. Its specificity for the alpha2-adrenoceptor, and relative selectivity for the alpha2A-adrenoceptor subtype (which is responsible for its sedative properties), provides for a more effective sedative and analgesic agent than clonidine. Dexmedetomidine is approximately eight times more "alhpa2-specific" than clonidine, with a ratio of alpha2 and alphal receptor affinity of 1,600:1. Dexmedetomidine has a distribution half-life more than twice as short as clonidine $(<5$ vs $11 \mathrm{~min})$, a more rapid clearance $\left(0.75 \mathrm{~L} \cdot \mathrm{hr}^{-1} \cdot \mathrm{kg}-1\right.$ vs 0.2 $\left.\mathrm{L} \cdot \mathrm{hr}^{-1} \cdot \mathrm{kg}^{-1}\right)$, and a terminal elimination half-life of two hours, compared to nine hours for clonidine.

A variety of perioperative effects of alpha2 agents have been studied. These include effects on the stress response to surgery; myocardial ischemia; sedation, anxiolysis, and analgesia; hemodynamics; prevention of delirium; and mitigation of drug withdrawal.

\section{Stress response}

Intraoperative clonidine $\left(7 \mu \mathrm{g} \cdot \mathrm{kg}^{-1} i v\right)$ has been shown to increase stroke volume, decrease heart rate, and decrease shivering after abdominal aortic aneurysm repair, while decreasing plasma norepinephrine, epinephrine, and vasopressin levels. ${ }^{3}$ Postoperatively, dexmedetomidine infused for sedation (0.2-0.25 $\left.\mu \mathrm{g} \cdot \mathrm{kg}^{-1} \cdot \mathrm{hr}^{-1}\right)$ decreased interleukin-6 levels and heart rate compared to propofol $\left(17-50 \mu \mathrm{g} \cdot \mathrm{kg}^{-1} \cdot \mathrm{hr}^{-1}\right){ }^{4}$

\section{Myocardial ischemia}

In patients undergoing coronary surgery, clonidine 5 $\mu \mathrm{g} \cdot \mathrm{kg}-\mathrm{l}$ resulted in decreased plasma catecholamines and fewer episodes of ST segment depression compared to placebo. ${ }^{5}$ In a quantitative systematic review of 3,646

From the Department of Anesthesia and Pediatrics, Stanford University, Stanford, California, USA.

Address correspondence to: Dr. Gregory B. Hammer, Department of Anesthesia, Stanford University Medical Center, 300 Pasteur Drive, Stanford, CA 94305, USA. Phone: 650-723-7835; Fax: 650-725-8544; E-mail: ham@stanford.edu 
patients undergoing major non-cardiac surgery, the use of beta blockers and alpha 2 agonists (clonidine, mivazerol) were associated with a decrease in ischemic events and death compared with no benefit from calciumchannel blockers and nitroglycerine. ${ }^{6}$ A meta-analysis of randomized, controlled trials including 3,395 patients having cardiac surgery revealed that alpha 2 agonists decreased myocardial ischemia. ${ }^{7}$

\section{Sedation, anxiolysis, analgesia}

One of the highest densities of alpha2-adrenoceptor has been detected in the pontine locus ceruleus (LC), a key source of noradrenergic innervation of the forebrain and an important modulator of vigilance. The sedative effects of alpha2-adrenoceptor activation have been attributed to the inhibition of this nucleus. After dexmedetomidine binds to alpha2-adrenoceptors in the LC, transmembrane signaling results in activation of an inwardly rectifying potassium channel facilitating a $\mathrm{K}+$ efflux and inhibition of voltage-gated $\mathrm{Ca}^{2+}$ channels. The resulting hyperpolarization decreases the firing rate of LC neurons and allows presynaptic inhibition of their terminals. Hyperpolarization of noradrenergic LC neurons appears to be a key factor in initiating the anesthetic mechanism of action of dexmedetomidine. $^{8}$

Dexmedetomidine infusion (loading dose of 1.0 $\mu \mathrm{g} \cdot \mathrm{kg}^{-1}$ over ten minutes, then $0.2-0.7 \mu \mathrm{g} \cdot \mathrm{kg}^{-1} \cdot \mathrm{hr}^{-1}$ ) after major surgery causes a reduction in the amount of propofol, midazolam, and morphine required for sedation and analgesia. ${ }^{9,10}$

\section{Hemodynamics}

Dexmedetomidine typically causes a decrease in heart rate and blood pressure during continuous infusion, commensurate with the reduction in plasma catecholamine levels. ${ }^{11}$ Hemodynamic parameters return to baseline within one hour of stopping the infusion. A transient increase in blood pressure may be seen with bolus dosing, due to stimulation of the alpha2 adrenergic receptors in the peripheral vasculature. ${ }^{12}$ This effect is overridden by the central hypotensive effects once the drug crosses the blood-brain barrier within several minutes. Reflex bradycardia may be observed in association with the acute increase in blood pressure during bolus dosing.

In summary, bolus dose infusions of dexmedetomidine (and high-dose infusions) cause a reduction in plasma catecholamines, an increase in systemic and pulmonary vascular resistances, and a decrease in stroke volume, heart rate, and blood pressure.

Lower dose continuous infusions of dexmedetomidine produce a decrease in plasma catecholamines, minimal change in vascular resistances, and mild reductions in heart rate and blood pressure.

\section{Delirium}

Postoperative delirium is common in adult patients after cardiac surgery. The surgical procedure and cardiopulmonary bypass effects have been implicated. Recently, however, off-pump cardiac surgery has become more common, but the incidence of postoperative delirium has not decreased significantly in these patients. The role of sedative and analgesic agents in postoperative delirium has been a subject of greater scrutiny. A recent study at Stanford compared postoperative sedation with dexmedetomidine to standard regimens of propofol and midazolam. Fentanyl was used for analgesia in all three groups. Delirium, as defined by DSM-IV criteria, occurred in 50\% of patients in the propofol and midazolam groups, but in only $2 \%$ of the patients sedated with dexmedetomidine. Delirium is much more difficult to define in infants and children but may be prevalent. The role of dexmedetomidine for postoperative sedation in the pediatric population remains to be defined.

\section{Drug withdrawal and rebound}

Clonidine has been used to mitigate or prevent as well as to treat signs of withdrawal from alcohol, opioids, and other drugs. ${ }^{13}$ Alpha2 adrenergic agonists probably play a primary role by binding to opioid and other receptors in the central nervous system. They also decrease the signs and symptoms of drug withdrawal by decreasing sympathetic outflow in the central nervous system, thereby diminishing tachycardia, sweating, and tremor. There are several reports in the literature describing the use of dexmedetomidine for the prevention and treatment of sings of withdrawal from midazolam, lorazepam, and opioids. ${ }^{14,15}$ Unlike morphine, dexmedetomidine does not cause hyperalgesia after cessation of long-term administration. ${ }^{16}$

\section{Dexmedetomidine: pediatric experience}

Few studies of dexmedetomidine have been done in children. The pharmacokinetics and pharmacodynamics of dexmedetomidine have been described in one recent study of 18 children between the ages of two and $18 \mathrm{yr}$. After beginning a ten-minute infusion, the onset of sedation was rapid and the effects were dissipated within one hour (Lerman, submitted for publication). There was a mild, dose-dependent reduction in heart rate and no respiratory depression was observed during infusions of 2, 4, and $6 \mu \mathrm{g} \cdot \mathrm{kg}^{-1} \cdot \mathrm{hr}^{-1}$. The volume of distribution was slightly larger and the plasma clearance was slightly prolonged compared 
with adults, resulting in a comparable terminal halflife. Several case reports describing the use of dexmedetomidine in infants and children have shown similar sedative, opioid-sparing, and hemodynamic effects as in adults.

\section{References}

1 Venn. Critical Care 2000; 4: 302

2 Ebert. Anesthesiology 2000; 93: 382.

3 Quintin. Br J Anaesth 1991; 66: 108.

4 Venn. Br J Anaesth 2001; 86: 650.

5 Dorman. JCVA 1993; 7: 386.

6 Stevens. Anesth Analg 2003; 97: 623.

7 Wijeysundera. Am J Med 2003; 114: 742.

8 Nelson. Anesthesiology 2003; 98: 428.

9 Venn. Anaesthesia 1999; 54: 1136.

10 Triltsch. Crit Care Med 2002; 30: 1007.

11 Talke. Anesth Analg 1997; 85: 1136.

12 Bloor. Anesthesiology 1992; 77: 1134.

13 Jasinski. Arch Gen Psychiatry 1985; 42: 1063.

14 Macciolo. Anesthesiology 2003; 98: 575.

15 Multz. Anesth Analg 2003; 96: 1054.

16 Davies. Anesth Analg 2003; 96: 195. 